\title{
Enhancing Transmission Capacity of Weighted Networks
}

\author{
Fei Shao* and Zinan Chang \\ School of Computer Engineering \\ Jinling Institute of Technology \\ Nanjing 211169, China \\ *shaofei@jit.edu.cn
}

\begin{abstract}
How to control the traffic congestion and improve the transmission capacity of weighted network is of great importance in real world networks. In some weighted networks, the weight is the geographic distance between nodes. The transmission capacity of weighted network is investigated according to different node delivery capability schemes. In the schemes that delivery capability of each node is proportional to its degree and proportional to its strength, the transmission capacity can be enhanced greatly after deleting some edges while the network integrity is still maintained. Simulations show that deleting edges according to their original weight is proved to be more effective. It is of great practical importance in designing the network topological structure.
\end{abstract}

Keywords: Weighted network, BBV network, delivery capability, transmission capacity

\section{Introduction}

The network topological structure such as small-world effect [1] and scale-free character [2] have been proved to have great influence on the traffic dynamics. To enhance the transmission capacity of complex network, such as the Internet, the power grid, and the airport networks is of great interest [3-6]. A basic assumption in these studies is that the network is unweighted network where edges between nodes are either present or not. Recently works reveal that many real world networks such as the scientific collaboration networks [7], the world-wide airport network [8] and the Internet [9] possess weighted feature that nodes interact with each other with varying strength. Lots of models have been presented to describe those weighted networks [10-12]. The BBV model [10], which is proposed to describe the weights reinforcement phenomenon triggered by the addition of new nodes, is the most widely used one.

Recent years have witnessed that enhancing the transmission capacity is gaining more and more concern of researchers. Some focus on designing more effective routing strategies [13-18] such as the Dijkstra shortest path [13] routing strategy, the efficient path [14] routing strategy. The Dijkstra shortest path [13] routing strategy is widely applied in lots of real world networks because its efficiency is the highest with delivery time being equal to the shortest path length. In some weighted networks, weights are statistical measures of tie strength which need to be reversed before applying Dijkstra shortest path algorithm to identify the shortest paths. In some other weighted networks, weights are the geographic distance which can directly apply the Dijkstra shortest path algorithm. Others are interested in changing the network topology structure [19-21] rather than designing routing strategies. It is proved that kicking out some edges linking to nodes with large betweenness can enhance network transmission capacity of scale-free networks[20]. 
Based on the idea, in this paper, we investigate the impact of deleting some edges in weighted networks on the transmission capacity. According to the different definitions of edge weight, we test the efficiency of our deleting edges strategies by checking the critical packet generating rate in three different node delivery capability schemes.

This paper is organized as follows. In section 2 we describe the BBV weighted network model, the traffic model and our deleting edges strategies, followed by the experimental simulations in section 3 . The conclusions are given in section 4 .

\section{Models}

A weighted adjacency matrix $\boldsymbol{W}$, whose elements $w_{i j}$ denote the weight of the edge between node $i$ and $j$, is often used to describe BBV weighted network. The generating of BBV weighted networks is as follows:[8, 22]

1) Starting from $N_{0}$ nodes fully connected by edges with weight $w_{0}$, a new node is added at each time step. The newly added node will be connected to $m$ different previously existing nodes with equal weight $w_{0}$ for every edge, choosing preferentially nodes according to the probability $\prod_{n \rightarrow i}=s_{i} / \sum_{l} s_{l}$, where $s_{i}$ is the node strength, $s_{i}=\sum_{j} w_{i j}$.

2) The creation of edge connecting to node $i$ will introduce variations of the weight of the other edges linked to node $i$, which is set to be proportional to the edge weights. If the total increase is $\delta$ ( $\delta_{i}=\delta$ for simplicity), we can get

$$
w_{i j}=w_{i j}+\Delta w_{i j}=w_{i j}+\delta * \frac{w_{i j}}{s_{i}}
$$

The transmission model can be described as follows:

1) All the nodes can generate packets with addresses of destination, receive packets and route the packets to their destinations according to the routing strategy.

2) At each time step $t, R$ packets are generated in the whole network and placed at the end of the queue.

3) At the same time, the first $C_{i}$ packets at the top of the queue of each node $i$ are forwarded one step toward their destinations.

4) The packets are removed from the network when they reach their destinations.

We give our deleting edges strategies as follows:

1) Define the weight of the edge connecting nodes $i$ and $j, W_{i j}$, in different way according to deleting edges strategies shown in Table 1.

\section{Table 1. The Definition Weight $W_{i j}$ of Different Deleting Edges Strategies}

\begin{tabular}{|l|l|l|l|}
\hline & DOW & DDP & DSP \\
\hline$W_{i j}$ & $w_{i j}$ & $k_{i}{ }^{*} k_{j}$ & $s_{i}{ }^{*} s_{j}$ \\
\hline
\end{tabular}

In table $1, W_{i j}$ is the definition weight which is different according to different definition. $w_{i j}$ is the original weight of the edge and $s_{i}$ is the strength of node $i$ as mentioned above. And $k_{i}$ is the degree of node $i$ which is the neighbors number of node $i$. 
2) Sort the edges by the definition weight $W_{i j}$ in decreasing order and delete the edge ranked first. If deleting an edge will cause the division of the whole network, we will keep it and go to deal with the edge ranked next.

3) Recalculate the definition weight $W_{i j}$ and repeat step 2 until all deletable edges are deleted. $\left(f_{\mathrm{e}}\right.$ is the fraction of deleted edges in all deletable edges. $f_{\mathrm{e}}=0$ means no edges are deleted which is the original network. $f_{\mathrm{e}}=1$ means all deletable edges are deleted which is $n-m+1$ edges are deleted.)

4) The Dijkstra shortest path routing strategy[13] is applied in the new network with using the original edge weight directly.

We utilize the RAN deleting edges strategy, where edges are deleted randomly, to check the validity of our strategies.

We also take advantage of the weighted betweenness $b_{w i}$ to estimate the possible packets passing through node which is defined as [23, 24]

$$
b_{w i}=\sum_{s, t} \frac{\sigma(s, i, t)}{\sigma(s, t)}
$$

$\sigma(s, i, t)$ is the number of paths under the given routing strategy between nodes $s$ and $t$ that pass through node $i$ and $\sigma(s, t)$ is the total number of paths under the given routing strategy between node $s$ and $t$.

In a weighted network with $n$ nodes, a certain packet will pass through the node $i$ with probability $b_{w i} / \sum_{j=1}^{n} b_{w j}$. The node $i$ will receive $R^{*} b_{w i} /(n *(n-1))$ packets at each time step. When the node $i$ receives more packets than its delivery capability, that is $R^{*} b_{w i} /(n *(n-1))>C_{i}$, network congestion occurs. So the critical packet generating rate $R_{\mathrm{c}}$, which can best reflect the transmission capacity of the network, is

$$
R_{c}=\min \left(C_{i} * n *(n-1) / b_{w i}\right)
$$

In unweighted networks, it is often assumed that all nodes have same delivery capability which is usually set as $C_{i}=1$ for simplicity $[14,25]$ (CCON stands for this scheme). And the node delivery capability is considered to be proportional to its degree $k_{i}$ (CDEG stands for this scheme) [6]. In weighted networks, we consider the other situation: the node delivery capability is proportional to its strength $s_{i}$ (CSTR stands for this scheme).

\section{Simulations and Analysis}

At first, we investigate the validity of our deleting edges strategies by checking the critical packet generating rate $R_{c}$ in three different node delivery capacity schemes in BBV network with $n=250, \delta=4, m=4$ and $w_{0}=1$. Simulation results are shown in figure 1.

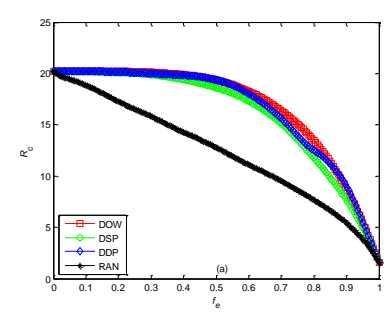

(a) CCON

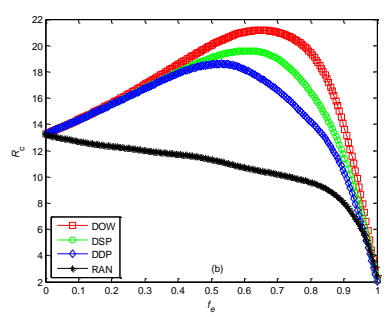

(b) CDEG

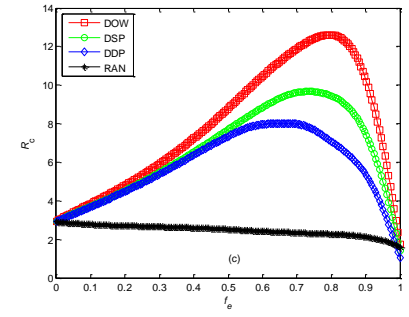

(c) CSTR

Figure 1. $R_{\mathrm{c}}$ VS $f_{\mathrm{e}}, n=250, \delta=4, m=4, w_{0}=1$ 
From figure 1, we can obtain that in the CCON scheme where all nodes have same delivery capability, deleting edges will result in the decline of the transmission capacity. However, when the node delivery capability is proportional to its degree or its strength, deleting some edges will enhance the transmission capacity greatly especially in the CSTR scheme. In the CDEG scheme, the maximum transmission capacity of the DOW strategy is $59.81 \%$ greater than in the original network while the DSP strategy and the DDP strategy is $48.06 \%$ and $40.62 \%$ correspondingly. In the CSTR scheme, the maximum transmission capacity of the DOW strategy is $368.78 \%$ greater than in the original network while the DSP strategy and the DDP strategy is $232.72 \%$ and $176.06 \%$ correspondingly. The maximum transmission capacity of the DOW strategy is better than the other two strategies (13.64\% higher than DDP and 7.94\% higher than DSP in the CDEG scheme, $69.81 \%$ higher than DDP and $40.89 \%$ higher than DSP in the CSTR scheme). The RAN deleting edges strategy cannot enhance the transmission capacity.

Then we change the weight parameter $\delta$ to check the impact of the weight on the critical packet generating rate $R_{\mathrm{C}}$ only in the CDEG scheme and the CSTR scheme where the deleting edges strategies works well. Simulation results are shown in figure 2.

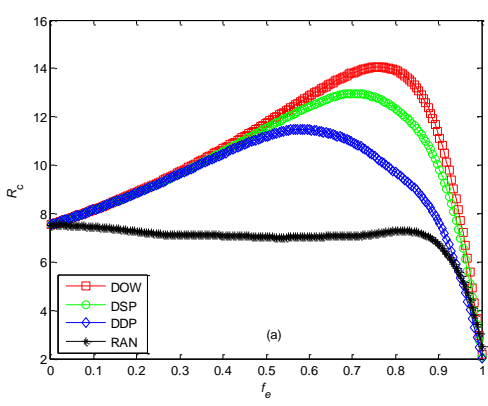

(a) $\delta=8, \mathrm{CDEG}$

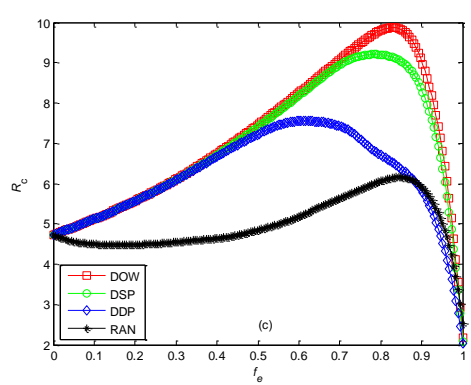

(c) $\delta=16$, CDEG

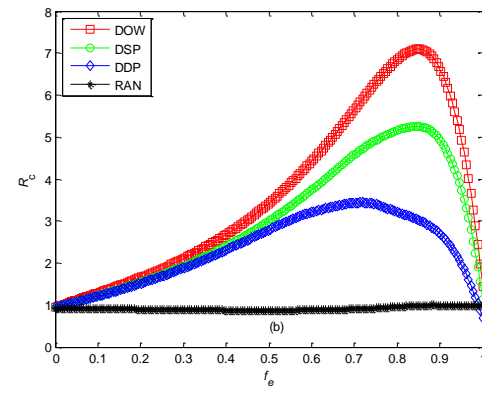

(b) $\delta=8$, CSTR

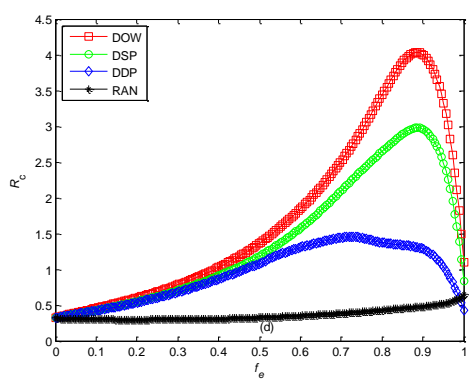

(d) $\delta=16$, CSTR

Figure 2. $R_{\mathrm{c}}$ VS Fe, $n=250, m=4, w_{0}=1$

Figure 2 also proves that deleting edges can enhance the transmission capacity in the CDEG scheme and the CSTR scheme. The enhancement of three deleting edges strategies on transmission capacity (the maximum the critical packet generating rate of each strategies VS the original one where $f_{\mathrm{e}}=0$ ) are shown in table 2 .

Table 2. The Enhancement of Three Deleting Edges Strategies

\begin{tabular}{|l|l|l|l|}
\hline & DSP & DDP & DOW \\
\hline$\delta=4$, CDEG & $40.62 \%$ & $48.06 \%$ & $59.81 \%$ \\
\hline$\delta=8$, CDEG & $52.56 \%$ & $72.27 \%$ & $86.74 \%$ \\
\hline$\delta=16$, & $59.76 \%$ & $94.80 \%$ & 109.23 \\
\hline
\end{tabular}




\begin{tabular}{|c|c|c|c|}
\hline & $\overline{\mathrm{DSP}}$ & $\overline{\text { DDP }}$ & DOW \\
\hline CDEG & & & $\%$ \\
\hline$\delta=4$, CSTR & $\%^{176.06}$ & $\%^{232.72}$ & $\%{ }^{368.78}$ \\
\hline$\delta=8$, CSTR & $\%^{270.72}$ & $\%^{466.28}$ & $\%^{666.70}$ \\
\hline $\begin{array}{r}\delta=16, \\
\text { CSTR }\end{array}$ & $\%^{358.15}$ & $\%^{842.78}$ & $\%^{1176.56}$ \\
\hline
\end{tabular}

From table 2, we can obtain that the total increase weight $\delta$ does affect the efficiency of our strategies. In the CDEG scheme and the CSTR scheme, the greater the weight $\delta$ is, the more effective our deleting edges strategies are, especially in the CSTR scheme.

The influence of the newly added edge number $m$ on our deleting edges strategies is shown in figure 3.

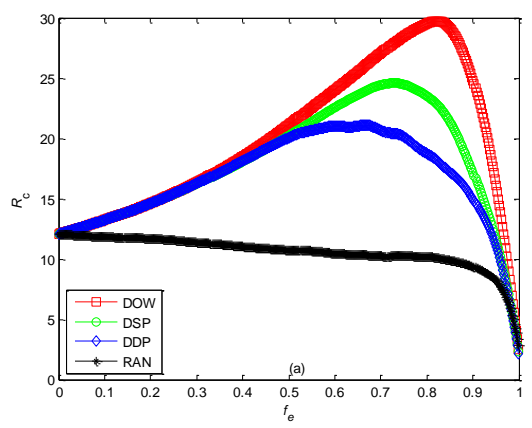

(a) CDEG

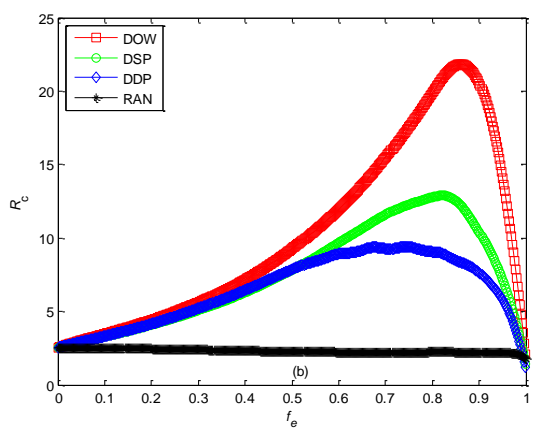

(b) CSTR

Figure 3. $R_{\mathrm{c}}$ VS $t_{\mathrm{e}}, n=250, \delta=4, m=8, w_{0}=1$

Figure 3 present the efficiency of our strategies in the CDEG scheme and the CSTR scheme as well. The enhancement of our strategies on transmission capacity is $75.05 \%, 103.53 \%$ and $146.29 \%$ in the CDEG scheme and $271.60 \%, 407.42 \%$ and $759.82 \%$ in the CSTR scheme which means the newly added edge number $m$ also affects the efficiency of our deleting edges strategies. Our strategies are more effective when more edges are added.

Then we check the impact of the total node number $n$ on our strategies. Simulation results are shown in figure 4.

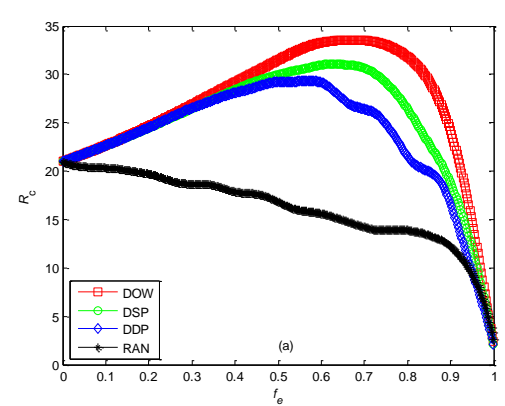

(a) CDEG

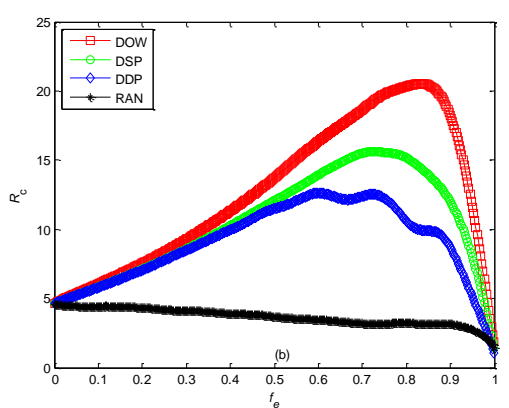

(b) CSTR

Figure 4. $R_{\mathrm{c}}$ VS Fe, $n=500, \delta=4, m=4, w_{0}=1$ 
The enhancement of our strategies on transmission capacity is $39.78 \%, 48.26 \%$ and $60.02 \%$ in the CDEG scheme and $174.53 \%, 239.08 \%$ and $345.98 \%$ in the CSTR scheme which means the total node number $n$ almost does not affect the efficiency of our deleting edges strategies.

From what has been mentioned above, we can come to the conclusion that in the CCON scheme where nodes have same delivery capability, our deleting edges strategies do not work. In the CDEG scheme and the CSTR scheme where the node delivery capability is proportional to its degree and its strength correspondingly, our deleting edges strategies can enhance the transmission capacity greatly especially in the CSTR scheme. And deleting edges according to their original weight is the most effective one.

The relationship between the average shortest lengths $L_{\mathrm{AVE}}$ and the total node number $n$ is reported in figure 6 .

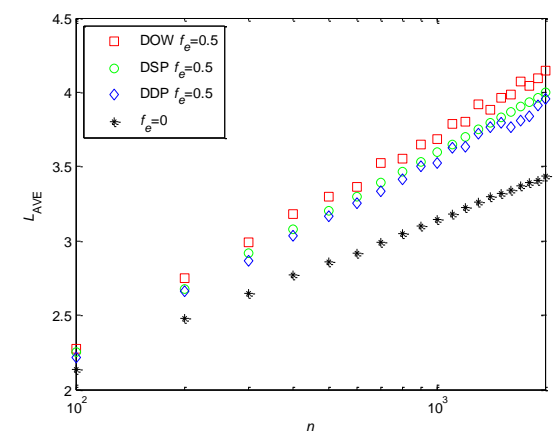

Figure 5. $L_{\mathrm{AVE}}$ VS $N, \Delta=4, M=4$ and $W_{0}=1$

Although after deleting 50\% deletable edges, the average shortest length of our strategies are higher than that of the original network, the small-world phenomenon $\left(L_{A V E} \propto \ln n\right.$ ) is still maintained. It means our deleting edges strategies are also of great practical importance in the field of enhancing transmission capacity of real world networks.

It is worth mentioning that deleting about half of deletable edges can enhance transmission capacity, but if all deletable edges are deleted, the transmission capacity will be reduced on the contrary.

\section{Conclusions}

Aiming at enhancing the transmission capacity of weighted networks, three deleting edges strategies are proposed. We check the critical packet generating rate in three different node delivery capability schemes after deleting some edges. If all nodes have same delivery capability, our deleting edges strategies does not work. However, when the node delivery capability is proportional to its degree or its strength, our deleting edges strategies can enhance the transmission capacity greatly. Both the total increase weight $\delta$ and the newly added edge number $m$ affect the efficiency of our deleting edges strategies. Our strategies are more effective when these two parameters are greater. The total node number $n$ almost does not affect the efficiency of our strategies. Deleting edges according to their original weight is the most effective one while deleting edges randomly will not enhance the transmission capability. The small-world phenomenon of average length is maintained while the whole network integrity is still protected. 


\section{Acknowledgements}

This work was partially supported by the National Natural Science Foundation of China (Grant No. 61373136, 61375121), the Natural Science Foundation of Jiangsu Province, China (Grant No. BK2012082), the Natural science fund for colleges and universities in Jiangsu Province, China (Grant No. 14KJB520012), the Research Foundation of Jinling Institute of Technology (Grant No. JIT-B-201406) and sponsored by Qing Lan Project and the Jiangsu Provincial Government Scholarship for Overseas Studies. The author also gratefully acknowledges the helpful comments and suggestions of the reviewers, which have improved the presentation.

\section{References}

[1] D.J. Watts and S.H. Strogatz, "Collective dynamics of 'small-world' networks", NATURE, (1998), pp. 393, 6684

[2] A.L. Barabási and R. Albert, "Emergence of scaling in random networks", SCIENCE, vol. 286, no. 5439, (1999).

[3] Z. Toroczkai and K.E. Bassler, "Network dynamics: Jamming is limited in scale-free systems", NATURE, vol. 428, no. 6984, (2004).

[4] A. Arenas, A. Díaz-Guilera and R. Guimerà, "Communication in networks with hierarchical branching", PHYSICAL REVIEW LETTERS, vol. 86, no. 14, (2001).

[5] G.Q. Zhang, S. Zhou, D. Wang, G. Yan, and G.Q. Zhang, "Enhancing network transmission capacity by efficiently allocating node capability, Physical A: Statistical Mechanics and its Applications", vol. 390, no. 2, (2011).

[6] L. Zhao, Y.C. Lai, K. Park and N. Ye, "Onset of traffic congestion in complex networks", PHYSICAL REVIEW E, vol. 71, no. 2, (2005).

[7] M.E.J. Newman, "Scientific collaboration networks, II. Shortest paths, weighted networks, and centrality", PHYSICAL REVIEW E, vol. 64, no. 1, (2001).

[8] A. Barrat, M. Barthelemy, R. Pastor-Satorras and A. Vespignani, "The architecture of complex weighted networks", Proceedings of the National Academy of Sciences of the United States of America, vol. 101, no. 11, (2004).

[9] R. Pastor-Satorras and A. Vespignani, "Evolution and structure of the Internet: A statistical physics approach", Cambridge University Press, Cambridge, (2007).

[10] A. Barrat, M. Barthélemy, and A. Vespignani, "Weighted evolving networks: coupling topology and weight dynamics", PHYSICAL REVIEW LETTERS, vol. 92, no. 22, (2004).

[11] S.H. Yook, H. Jeong, A.L. Barabási and Y. Tu, "Weighted evolving networks", PHYSICAL REVIEW LETTERS, vol. 86, no. 25, (2001).

[12] T. Antal and P.L. Krapivsky, "Weight-driven growing networks", PHYSICAL REVIEW E, vol. 71, no. $2,(\mathbf{2 0 0 5})$

[13] E.W. Dijkstra, "A note on two problems in connexion with graphs", Numerische mathematik, vol. 1, no. $1,(1959)$.

[14] G. Yan, T. Zhou, B. Hu, Z.Q. Fu and B.H. Wang, "Efficient routing on complex networks", PHYSICAL REVIEW E, vol. 73, no. 4, (2006).

[15] X. Ling, M.B. Hu, R. Jiang and Q.S. Wu, "Global dynamic routing for scale-free networks", PHYSICAL REVIEW E, vol. 81, no. 1, (2010).

[16] T. Zhou, "Mixing navigation on networks, Physica A: Statistical Mechanics and its Applications", vol. 387, no. 12, (2008).

[17] M.B. Hu, R. Jiang, Y.H. Wu, W.X. Wang and Q.S. Wu, "Routing on a weighted scale-free network, Physica A: Statistical Mechanics and its Applications", vol. 387, (2008), pp. 19-20.

[18] C.Y. Yin, B.H. Wang, W.X. Wang, T. Zhou and H.J. Yang, "Efficient routing on scale-free networks based on local information", PHYSICS LETTERS A, vol. 351, (2006), pp. 4-5.

[19] R. Guimerà, A. Díaz-Guilera, F. Vega-Redondo, A. Cabrales, and A. Arenas, "Optimal network topologies for local search with congestion”, PHYSICAL REVIEW LETTERS, vol. 89, no. 24, (2002).

[20] G.Q. Zhang, D. Wang, and G.J. Li, "Enhancing the transmission efficiency by edge deletion in scale-free networks", PHYSICAL REVIEW E, vol. 76, no. 1, (2007).

[21] G.Q. Zhang, "On cost-effective communication network designing”, EUROPHYSICS LETTERS, vol. 89, no. 3, (2010).

[22] A. Barrat, M. Barthélemy and A. Vespignani, "Modeling the evolution of weighted networks", PHYSICAL REVIEW E, vol. 70, no. 6, (2004).

[23] L.C. Freeman, "A set of measures of centrality based on betweenness", Sociometry, vol. 40, no. 1, (1977). 
[24] T. Opsahl, F. Agneessens and J. Skvoretz, "Node centrality in weighted networks: Generalizing degree and shortest paths", Social Networks, vol. 32, no. 3, (2010).

[25] B. Danila, Y. Yu, J.A. Marsh and K.E. Bassler, "Optimal transport on complex networks", PHYSICAL REVIEW E, vol. 74, no. 4, (2006).

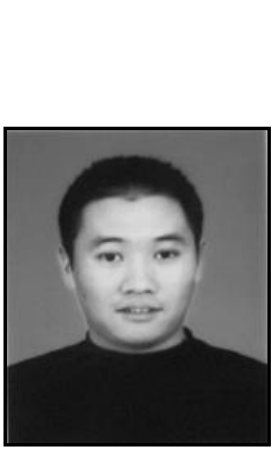

\section{Authors}

Fei Shao, He received the Bachelor Degree in Computer Application from Jiangsu University of Science and Technology in 1998, Master degree in Computer Application Technology from Nanjing University of Technology in 2003, and Ph. D Degree in Information Security from Nanjing University of Posts \& Telecommunications in 2013. From 2010 to now, he is an associate professor at the School of Computer Engineering, Jinling Institute of Technology and working for Jiangsu Information Analysis Engineering Laboratory. His current research interests include information security and complex dynamical networks.

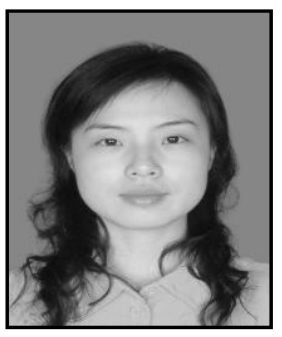

Zinan Chang, She received the Bachelor Degree in Computational Mathematics from Nanjing Normal University in 2000, Master degree in Computational Mathematics from Nanjing Normal University in 2006. From 2006 to now, she is a lecture at the School of Computer Engineering, Jinling Institute of Technology and working for Jiangsu Information Analysis Engineering Laboratory. Her current research interests include information security and complex dynamical networks. 